\title{
Pertinencia curricular de la carrera de Software en la Universidad Estatal de Bolívar
}

Curricular relevance of the software career at the Bolivar' State University

\author{
Galuth Irene García Camacho. 1, Maricela Araceli Espín Morejón. 2, Mónica \\ Elizabeth Bonilla Manobanda. ${ }^{3} \&$ Darwin Paul Carrión Buenaño. ${ }^{4}$
}

Recibido: 06-10-2021 / Revisado: 18 -10-2021 /Aceptado: 04-11-2021/ Publicado: 05-12-2021

\begin{abstract}
DOI: https://doi.org/10.33262/concienciadigital.v4i4.2.1933

Introduction. For some years in Ecuador evaluations of higher education have been carried out as a strategy to guarantee its quality, being the relevance of the careers that plays a crucial role in the evaluation carried out by the Council for the Assurance of the Quality of Education Superior (CACES). Objective. Determine the relevance of the software career at the State University of Bolívar (UEB) by studying its curriculum. Methodology. The research was qualitative and quantitative, retrospective and descriptive, an analysis was made in six dimensions, such as: prospective study of the career, contextual analysis, academic offer, demand, follow-up of graduates and employability through bibliographic review and application of surveys validated by specialists. Results. The results reflected that the Software career curriculum has several positive aspects; In the first instance, the knowledge and learning that allow to solve the

1 Universidad Estatal de Bolívar. Carrera de Software, Guaranda, Bolívar, Ecuador. ggarcia@ueb.edu.ec ORCID https://orcid.org/ 0000-0001-8692-4017

2 Universidad Estatal de Bolívar. Carrera de Software, Guaranda, Bolívar, Ecuador. mespin@ueb.edu.ec ORCID https://orcid.org/ 0000-0002-7652-5490

3 Universidad Estatal de Bolívar. Carrera de Software, Guaranda, Bolívar, Ecuador. mbonilla@ueb.edu.ec ORCID https://orcid.org/ 0000-0002-6268-3682

4 Universidad Estatal de Bolívar. Carrera de Software, Guaranda, Bolívar, Ecuador. dcarrion@ueb.edu.ec ORCID https://orcid.org/ 0000-0002-7754-3826
\end{abstract}


actions of future engineers are aligned to the objectives of the National Development Plan, the acceptance of the Software career by high school students and the criteria of graduates in the same branch of specialization as well as the opinion of potential employers were nuances that provided an important input for the curricular readjustment of the software career. Conclusion. The current software career at the UEB is relevant, but it is important to make some updates to its curriculum so that it continues to meet the level of satisfaction with respect to the skills that students acquire during their academic training and their impact on the sector professional.

Keywords: relevance, software, higher education, competences.

\section{Resumen}

Introducción. Desde hace algunos años en el Ecuador se realizan evaluaciones de la educación superior como estrategia para garantizar su calidad, siendo la pertinencia de las carreras aquella que juega un papel crucial en la evaluación realizada por el Consejo de Aseguramiento de la Calidad de la Educación Superior (CACES). Objetivo. Determinar la pertinencia de la carrera de software en la Universidad Estatal de Bolívar (UEB) mediante el estudio a su currículo. Metodología. La investigación fue cualitativa y cuantitativa, de tipo documental y descriptiva se hizo un análisis en seis dimensiones, tales como: estudio prospectivo de la carrera, análisis contextual, oferta académica, demanda, seguimiento a graduados y empleabilidad mediante revisión bibliográfica y la aplicación de encuestas validadas por pares expertos para asegurar su confiabilidad. Resultados. Los resultados reflejaron que el currículo de la carrera de Software tiene varios aspectos positivos; en primera instancia, los conocimientos y aprendizajes que permiten solventar el accionar de los futuros ingenieros se encuentran alineados a los objetivos del Plan Nacional de Desarrollo, la aceptación de la carrera de software por los estudiantes de bachillerato y el criterio de los graduados en la misma rama de especialización así como la opinión de los potenciales empleadores fueron matices que brindaron un insumo importante para la readecuación curricular de la carrera de Software. Conclusión. La actual carrera de software en la UEB es pertinente, pero es importante realizar algunas actualizaciones a su currículo para que continúe encaminada a cumplir con el nivel de satisfacción con respecto a las competencias que los estudiantes adquieran durante su formación académica y su impacto en el sector profesional.

Palabras clave: pertinencia, software, educación superior, competencias.

\section{Introducción}

La Organización de las Naciones Unidas para la Educación, la Ciencia y la Cultura (UNESCO, 1995, p. 8) indica que la pertinencia se considera:

"primordialmente, en función de su cometido y su puesto en la sociedad, de sus funciones con respecto a la enseñanza, la investigación y los servicios conexos, y 
de sus nexos con el mundo del trabajo en sentido amplio, con el Estado y la financiación pública y sus interacciones con otros niveles y formas de educación.”.

La pertinencia de la Educación Superior debe evaluarse en función de la adecuación de lo que la sociedad espera de las instituciones y lo que éstas hacen. Para ello se requiere normas éticas, imparcialidad política, capacidad crítica y, al mismo tiempo, una mejor articulación con los problemas de la sociedad y el mundo del trabajo, fundando las orientaciones a largo plazo en objetivos y necesidades sociales, comprendidos el respeto de las culturas y la protección del medio ambiente. (UNESCO, 1998, pp. 104-105).

La Constitución de la República del Ecuador en el Art 351 determina que: "El sistema de educación superior estará articulado al sistema nacional de educación superior y al Plan nacional de Desarrollo; ....se regirá por los principios de calidad, pertinencia, integralidad, autodeterminación para la producción del pensamiento y conocimiento....."Existen amplios consensos acerca de la necesidad de mejorar la educación superior a través de la evaluación, que se viene realizando en dos instancias, la evaluación institucional y la evaluación de carreras de grado y programas de posgrado. De acuerdo con lo que indica el Art 94 de la Ley Orgánica de Educación Superior (LOES), la evaluación de la calidad es un proceso que permitirá diagnosticar las condiciones en que se encuentra una institución, carrera o programa académico, de tal manera que sus resultados sirvan para mejorar la carrera o programa en cuestión (Asamblea Nacional de la República del Ecuador, 2010).

El Consejo de Aseguramiento de la Calidad de la Educación Superior ([CACES], 2017), respecto al principio de pertinencia, indica que es fundamental que la oferta de grado considere la planificación nacional y la política pública en educación superior, así también la normativa relacionada. En particular, para la revisión de la pertinencia de carreras, estos estándares consideran las características académicas del programa que respondan a los requerimientos del entorno, particularmente, que contribuyan a la planificación nacional para el desarrollo y a la reducción de brechas en sectores prioritarios y emergentes.

La universidad actual se enfrenta al desafío de insertarse en un mundo globalizado con nuevas exigencias relacionadas a la sociedad del conocimiento. Se requiere una universidad pertinente que incorpore un valor agregado a la experiencia de sus estudiantes y que responda a las necesidades de los actores sociales y productivos del entorno (Tamayo \& Cerda, 2017).

En este sentido, México es uno de los países latinoamericanos que más ha avanzado en materia de educación superior. Tienen una Asociación Nacional de Universidades e Instituciones de Educación Superior ([ANUIES], s.f.), que participa en la formulación de programas, planes y políticas, y la creación de organismos que se orientan al desarrollo de la educación superior. Promueven el mejoramiento integral de la docencia, la investigación y la extensión universitaria, agremiando a las principales instituciones, con 
un total de 180 universidades e instituciones de educación superior, tanto públicas como privadas. Además ha puesto énfasis en la elaboración de estudios de pertinencia, y un ejemplo de ello es la Universidad Autónoma de Yucatán ([UADY], 2012), que emitió una guía para elaboración de estudios de pertinencia social, factibilidad y estado del arte para la creación, modificación y liquidación de programas educativos.

En este contexto, el presente documento muestra el estudio realizado en el periodo académico 2020-2020 por 4 miembros de la comisión de rediseño de la carrera de software, con el propósito de determinar la pertinencia del currículo ofertado por la UEB en la ciudad de Guaranda, a fin de mejorar su plan de estudios a través de un análisis y readecuación de los componentes educativos, que permita establecer la congruencia entre la oferta educativa y las necesidades sociales.

\section{Metodología}

El diseño de la investigación fue cualitativa y cuantitativa, comprendió una fase de investigación documental y una descriptiva aplicando los métodos inductivo y deductivo, recabando y analizando datos en documentos de instituciones públicas, privadas y educativas del Ecuador.

Fase Documental: consistió en una revisión bibliográfica en documentos de entidades gubernamentales y educativas que permitieron conocer la prospectiva de la carrera, hacer su análisis contextual, identificar la oferta y demanda de esta así como realizar un análisis retrospectivo sobre los graduados en el área; es importante mencionar que la carrera de Software entró en vigencia en el año 2017, por lo que al momento del estudio aún no tenía una cohorte de titulados, para conocer las experiencias de los graduados se analizaron los resultados obtenidos en el seguimiento a graduados de la carrera de Sistemas realizado en el año 2020, considerando también que la carrera de Software nació como rediseño de la carrera de Sistemas.

Fase Descriptiva: se realizó la recolección información de fuentes primarias y el análisis de la demanda de la carrera así como la empleabilidad de los futuros Ingenieros en software, mediante la aplicación de encuestas a empleadores y estudiantes de bachillerato en la provincia Bolívar, el instrumento de investigación fue validado por pares expertos para asegurar su confiabilidad.

La encuesta al sector estudiantil se realizó considerando los cantones más notables de la provincia Bolívar, eligiendo instituciones educativas que cuentan con bachillerato, haciendo un total de 824 encuestados entre estudiantes de segundo y tercer año.

La muestra de estudio en el sector de empleadores se determinó mediante un análisis crítico del universo, construyendo una lista de 91 organizaciones tanto públicas como privadas de los cantones más notables de la provincia Bolívar, considerando el grado de importancia de la institución en la provincia y/o cantón, aspectos sociales, técnicos y económicos, así como la actual emergencia sanitaria por el COVID-19 que limitó la movilización y acceso a diferentes entidades. Para el cálculo de la muestra se utilizó la 
ecuación 1 (Bologna, 2018), con un nivel de confianza del 95\% para que la cantidad de encuestas sea confiable; se usó el valor de p=0,5 asumiendo que el $50 \%$ de la población cumple con la característica de estudio.

$$
\mathbf{n}=\frac{\mathrm{N} * Z^{2} * \mathrm{p} * \mathrm{q}}{e^{2} *(N-1)+Z^{2} * p * q} \quad \text { Ecuación 1. Fórmula del Muestreo }
$$

Las encuestas fueron aplicadas a través de formularios en línea.

\section{Resultados y Discusión}

En este proceso investigativo se evaluó la pertinencia del currículo de la carrera de Software de la UEB, considerando aspectos tales como: prospectiva de la carrera, análisis contextual, oferta y demanda de la carrera de Software, seguimiento a graduados de una carrera del área y análisis de empleabilidad.

Como resultado del estudio prospectivo de la carrera de Software realizado por la dirección de planeamiento y aseguramiento de la calidad de la UEB (2020) se dio a conocer que existe una necesidad creciente de desarrolladores de software talentosos en todas las industrias. A medida que avanza la tecnología, la capacidad de crear software de calidad considerando el diseño, el desarrollo, la seguridad y el mantenimiento se busca entre todo tipo de empresas, desde las finanzas y la banca hasta la atención médica y la seguridad nacional. Esto después de un análisis a 54 variables, tales como: Resultados de investigación, oferta académica con integración cultural, talento humano competente, Infraestructura disponible, sistema de apoyo estudiantil, docentes, visión institucional prospectiva, laboratorios especializados, espacios de interacción con la sociedad representación estudiantil, bibliotecas, infraestructura nueva, modernización de los procesos administrativos financieros, Pocos recursos de autogestión, gestión administrativa financiera ineficiente, mallas curriculares desactualizadas, Poca articulación de las actividades sustantivas, Limitaciones para la investigación, Débil sistema de seguimiento a graduado, infraestructura con deficiencias, débil programa de fortalecimiento de las capacidades de talento humano, recursos físicos y tecnológicos limitados, débil gestión del talento humano, insuficiente ejecución de actividades de formación cultural, problemáticas de corrupción y acoso, débil sistema de acompañamiento estudiantil, ausencia de un sistema de gestión documental y de información, puestos docentes inadecuados, desarticulación en la planificación institucional, débiles procesos de vinculación del personal docente, desarticulación del trabajo con entidades externas, bajo porcentaje de docentes titulares, desarticulación entre la generación del conocimiento, construcción del saber y la excelencia académica, debilidad de la extensión de San Miguel, estructura orgánico funcional burocratizada, débil cultura organizacional, ausencia de un sistema de aseguramiento de la calidad, redes de investigación, localización geográfica, posicionamiento en las entidades que prestan apoyo a la investigación, empresa pública, convenios interinstitucionales, diversidad de actores externos de la sociedad, relación con los GAD's, estructura organizativa, 
mejoramiento profesional docente, déficit presupuestario, ausencia en la definición de gasto en $\mathrm{I}+\mathrm{D}+\mathrm{i}$, poco desarrollo económico de la provincia, poca estabilidad en el direccionamiento del Sistema Regional de Indicadores Estandarizados de Convivencia y Seguridad Ciudadana (SES), problemática estructural de la educación, desarticulación con los entes rectores, desarticulación con los problemas de la sociedad, débil respuesta ante la coyuntura mundial.

Por otra parte tras realizar la evaluación de 64 escenarios se ha centrado el análisis en los que tienen mayor probabilidad de ocurrencia, teniendo 6 que se destacan como se detalla a continuación:

- Se verifica que, dentro de los escenarios, el que mayor peso tiene es el denominado: La universidad entre las 500 mejores de Latinoamérica, mismo que tiene una valoración de 30 puntos en Institucionalizar la mejora continua.

- En el segundo criterio: fortalecer la función de investigación tienen un puntaje de 15 puntos, la universidad entre las 500 mejores de Latinoamérica y el escenario tendencial.

- En el tercer criterio: fortalecer la función de docencia el mayor puntaje de 20 puntos se encuentra en el escenario real.

- El cuarto criterio: fortalecer la función de vinculación tienen un puntaje de 25 puntos en el escenario tendencial y el real.

- En el quinto criterio: formar profesionales competentes tienen un puntaje de 25 puntos, en el escenario tendencial y el real.

- En el sexto criterio: optimizar los procesos de gestión de talento humano tienen un puntaje de 20 puntos en el escenario real.

Mostrando que en la Universidad Estatal de Bolívar actualmente está en un escenario real.

El análisis contextual permitió conocer las bondades de la región de estudio, la zona 5 está integrada por las provincias de Guayas, Los Ríos, Santa Elena, Bolívar y Galápagos, e incluye 48 cantones y 72 parroquias. Entre los cultivos más representativos de la Zona 5 se encuentran el banano, el cacao, el café, arroz, en las provincias Guayas y Los Ríos; la caña de azúcar y maíz en las provincias de Guayas, Los Ríos y Bolívar y frutas tropicales en toda la zona. Una importante característica de esta zona es la variedad de ecosistemas que posee debido a que está compuesta por las tres regiones naturales: costa, sierra e insular. En lo turístico destacan el parque nacional Galápagos, el bosque protector cordillera Chongón Colonche, la fauna marino-costera puntilla de Santa Elena, los manglares de Churute del Guayas, el bosque protector Cascha Totoras y parte de la reserva faunística Chimborazo, en la provincia de Bolívar (Secretaría Técnica del Sistema Nacional Descentralizado de Planificación Participativa [SENPLADES], 2017).

Con el análisis contextual también se evidenció que el currículo de la Carrera de Software se encuentra alineado con los objetivos del Plan Nacional de Desarrollo (PND), en sus tres ejes: Eje 1 - objetivo 1: Garantizar una vida digna con iguales oportunidades para todas las personas; en donde el profesional de la carrera de Software será capaz de 
proponer soluciones tecnológicas que permita simplificar los procesos y procedimientos administrativos de las organizaciones fomentando el desarrollo nacional, favoreciendo a la política 6: garantizar el derecho a la salud, la educación y al cuidado integral durante el ciclo de vida, bajo criterios de accesibilidad, calidad y pertinencia territorial y cultural. En el Eje 2 -objetivo 5: Impulsar la productividad y competitividad para el crecimiento económico sostenible de manera redistributiva y solidaria; en donde el profesional de la carrera de Software estará en capacidad de elaborar y ejecutar proyectos de investigación y/o vinculación que respondan a las necesidades tecnologías de las organizaciones, impulsando prioritariamente a las actividades agropecuarias, agroindustriales, turísticas y de servicio de la zona 5, permitiendo la participación activa de los actores a través de soluciones tecnológicas, beneficiando a las políticas 6 y 8: promover la investigación, la formación, la capacitación, el desarrollo y la transferencia tecnológica, la innovación y el emprendimiento, la protección de la propiedad intelectual, para impulsar el cambio de la matriz productiva mediante la vinculación entre el sector público, productivo y las universidades; así como también, fomentar la producción nacional con responsabilidad social y ambiental, potenciando el manejo eficiente de los recursos naturales y el uso de tecnologías duraderas y ambientalmente limpias, para garantizar el abastecimiento de bienes y servicios de calidad. En el Eje 3 - objetivo 9: Garantizar la soberanía y la paz, y posicionar estratégicamente al país en la región y el mundo; en donde el profesional de la carrera de Software será capaz de implementar aplicaciones informáticas con el uso de estándares internacionales que permitan dinamizar los procesos involucrados en los servicios públicos y privados del país, favoreciendo a la política 3: al crear y fortalecer los vínculos políticos, sociales, económicos, turísticos, ambientales, académicos y culturales, y las líneas de cooperación para la investigación, innovación y transferencia tecnológica con socios estratégicos de Ecuador.

En relación con la oferta académica, la provincia de Bolívar cuenta con 21 carreras en estado vigente, todas impartidas por la Universidad Estatal de Bolívar y representando a nivel nacional el 1,07\% de las carreras vigentes y el 13,73\% de las carreras de la zona de planificación 5. La provincia de la zona 5 con mayor representatividad de la oferta vigente es los Ríos con el 40,52\% de las carreras, le sigue Guayas (excepto los cantones de Guayaquil, Samborondón y Durán), que posee el 28,10\% y en tercer lugar Santa Elena con el 16,34\% de esta (Secretaría de Educación Superior, Ciencia, Tecnología e Innovación [SNIESE], 2020). La Carrera de Software es ofertada sólo en dos universidades de la zona 5 en modalidad presencial, ubicadas en la región costa, por lo que es evidente que la Universidad Estatal de Bolívar sería un referente en esta carrera en la región sierra para la región de estudio. Así también se evidenció que de los 10 campos de conocimiento el campo Tecnologías de la Información y Comunicación se posiciona en un quito lugar con un $11,9 \%$, este resultado revela la baja oferta de carreras en el área informática, dejando clara la pertinencia de la actual oferta académica.

Como resultado derivado del análisis al seguimiento a graduados de la carrera de sistemas realizado en el año 2020, se conoció que el 63,34\% de los profesionales se encontraban 
laborando, de los cuales el $80 \%$ se desempeñan en su perfil profesional; un 54,29\% lo hacen en instituciones públicas, seguidas de las instituciones comerciales y académicas de nivel medio con un 14,29\% para cada una, los demás se encontraban ejerciendo su profesión en el sector productivo, investigación y educación superior. Las funciones dentro de las instituciones van desde el mando medio, operativo, ejecutivo y en un menor porcentaje quienes son gerentes propietarios de sus propios negocios; la relación laboral más representativa es la de contrato con un 68,57\%, seguida del nombramiento con $22,86 \%$ y finalmente como empleadores con $8,57 \%$, en su mayoría a tiempo completo. Con respecto al aporte de su formación académica recibida en la Escuela de Sistemas de la UEB en su desempeño profesional el 50\% consideró que ha sido en un nivel medio y un $36 \%$ en un nivel alto, lo que evidenció que las competencias adquiridas han tenido un impacto positivo en su desenvolvimiento laboral; además existieron recomendaciones para reforzar algunas áreas de especialidad, tales como: base de datos, seguridad informática, programación web, redes y comunicaciones.

Con respecto a la demanda de la carrera de software, las estadísticas presentadas en el archivo maestro de instituciones educativas en el Ecuador (Ministerio de Educación, 2019), dieron a conocer la existencia de 420 entidades educativas en la provincia entre urbanas y rurales, de las cuales 83 tiene bachillerato con un aproximado de 11.249 estudiantes cursando primero, segundo y tercero bachillerato, equivalente al $31,81 \%$ de la población estudiantil quienes serían los potenciales candidatos en acceder a la instrucción universitaria.

Por otra parte, el análisis descriptivo de la educación superior en el Ecuador realizado en enero del 2021 por la Dirección de Planeamiento y Aseguramiento de la Calidad de la UEB evidenció el aumento de la brecha de acceso a la carrera de Software, pues en el primero periodo del año 2019 se tuvo una brecha de 311 cupos no cubiertos, la cual ascendió a 419 para el primer periodo del año 2020. Así como también se constató que la Carrera de Software se encuentra entre las 15 carreras más demandadas dentro de la ubicación nacional de la oferta académica de la UEB en cada proceso desde el 2019 al 2020 (Goyes et al., 2021).

Como resultados de la encuesta aplicada al sector estudiantil se obtuvo que:

- El 69,90\% de encuestados tienen un título de bachillerato general unificado, el mismo que tiene como ventaja poder escoger cualquier carrera universitaria previo al examen de educación superior.

- Un 89,20\% de estudiantes de las instituciones de educación media desean continuar sus estudios y alcanzar un título de grado acorde a las necesidades en las que vivimos. Además, manifestaron que el principal factor para elegir una carrera de educación superior es la facilidad de ser aceptados y la posibilidad de emplearse al terminar la carrera.

- El 54,37\% de bachilleres de la provincia consideraron que no existe la suficiente oferta académica acorde al mundo digital en el que vivimos, por lo que se ve 
necesario continuar e incorporar carreras en el área tecnológica para evitar la salida de los estudiantes a otras provincias.

- En relación con el tipo de estudio de tercer nivel que desearían cursar el 74,39\% se inclina por estudiar una ingeniería, esto se debe a que la mayoría de los jóvenes tiene una idea de estudios netamente técnica. El 33,01\% desea continuar sus estudios superiores en una carrera técnica - tecnológica como es la Ingeniería en Software, al ser una carrera netamente especializada en computadores y que les permitirá alcanzar un empleo a corto plazo.

- Considerando las preferencias por la institución de educación superior en la que le gustaría continuar con sus estudios, un $25,85 \%$ se inclina por la Universidad Estatal de Bolívar, siendo un porcentaje aceptable, considerando que el porcentaje restante se encuentra distribuido en otras instituciones cercanas. Sin embargo, el $53,03 \%$ de estudiantes de educación media no están dispuestos a salir de su provincia para estudiar la carrera de su elección, esto por causa de factores sociales, económicos y culturales entre los más importantes.

- En una última pregunta los estudiantes aportaron libremente con sus comentarios, un gran porcentaje de encuestados felicitaron el accionar con este instrumento, pues con sus opiniones permiten mejorar la oferta educativa de la Universidad Estatal de Bolívar; entre los comentarios más relevantes están: que se den charlas del sistema educativo universitario en los colegios, se abran más carreras del área informática, se creen más carreras para que los jóvenes de la provincia no tengan que dejar sus hogares.

Con relación al análisis de empleabilidad para los profesionales de la carrera de software, se obtuvo como resultado que se debía encuestar a 50 instituciones entre públicas y privadas, de un total depurado de 91 instituciones, esto permitió conocer la percepción de los empleadores acerca del profesional en el área de programación y el grado de aceptación de los graduados en la carrera de Software de la UEB para su inserción en el ámbito laboral. La encuesta a empleadores arrojó los siguientes resultados:

La figura 1 muestra los profesionales graduados en las carreras ofertadas por la Facultad de Ciencias Administrativas, Gestión Empresarial e Informática (FaGEI) que laboran en diferentes instituciones país. 


\section{Figura 1}

Profesionales graduados en la UEB que laboran en diferentes instituciones

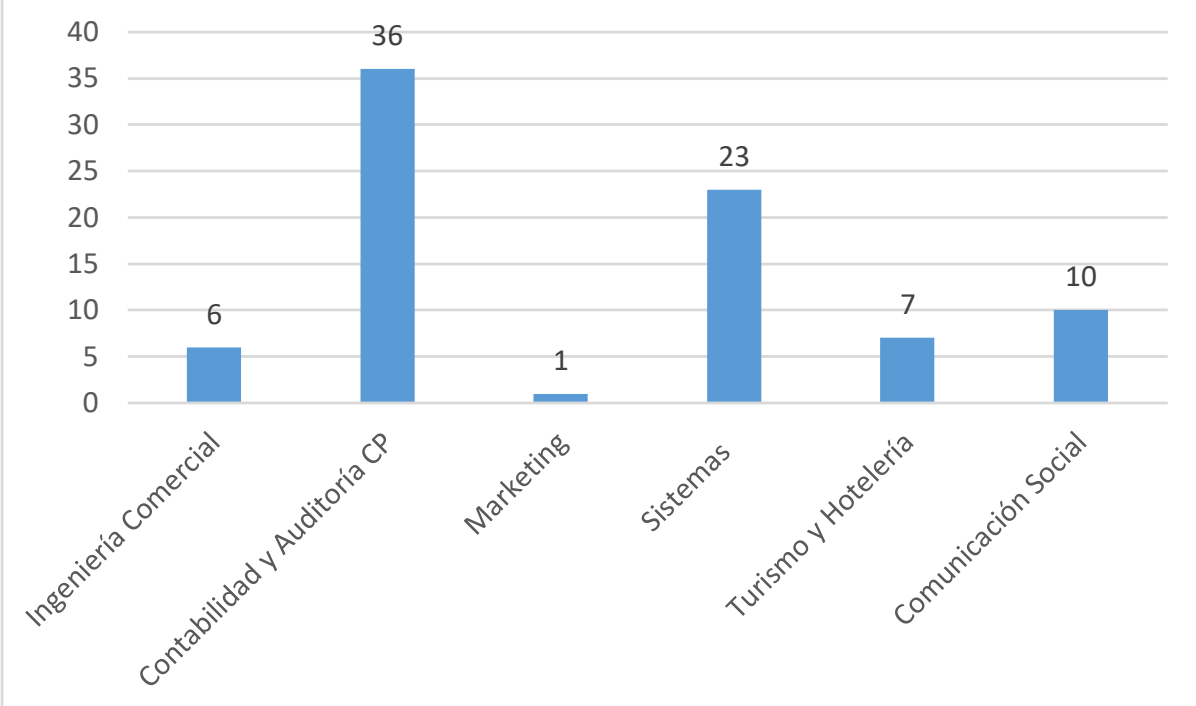

Fuente: Encuesta a empleadores, 2020

Se evidencia que los profesionales graduados en diferentes carreras de la FaCAGEI, se encuentran laborando en varias instituciones públicas y privadas, en alto porcentaje especialistas en Contabilidad y Auditoría seguidos por los Ingenieros en Sistemas que se encargan del soporte y mantenimiento de hardware y redes.

En la figura 2 se puede observar la necesidad de automatización de procesos en las instituciones.

\section{Figura 2}

Nivel de automatización de procesos en las instituciones

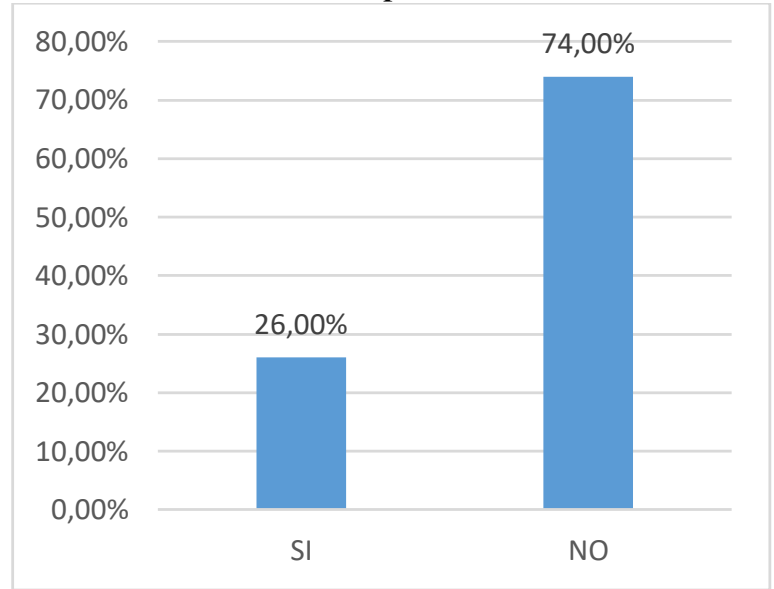

Fuente: Encuesta a empleadores, 2020

Un alto porcentaje de encuestados manifiestan no tener sistemas a medida para sus tareas cotidianas, lo que conlleva un bajo rendimiento de sus empleados provocando insatisfacción en sus clientes.

En la figura 3 se refleja la necesidad de automatizar los procesos de la empresa o institución en un corto plazo. 


\section{Figura 3}

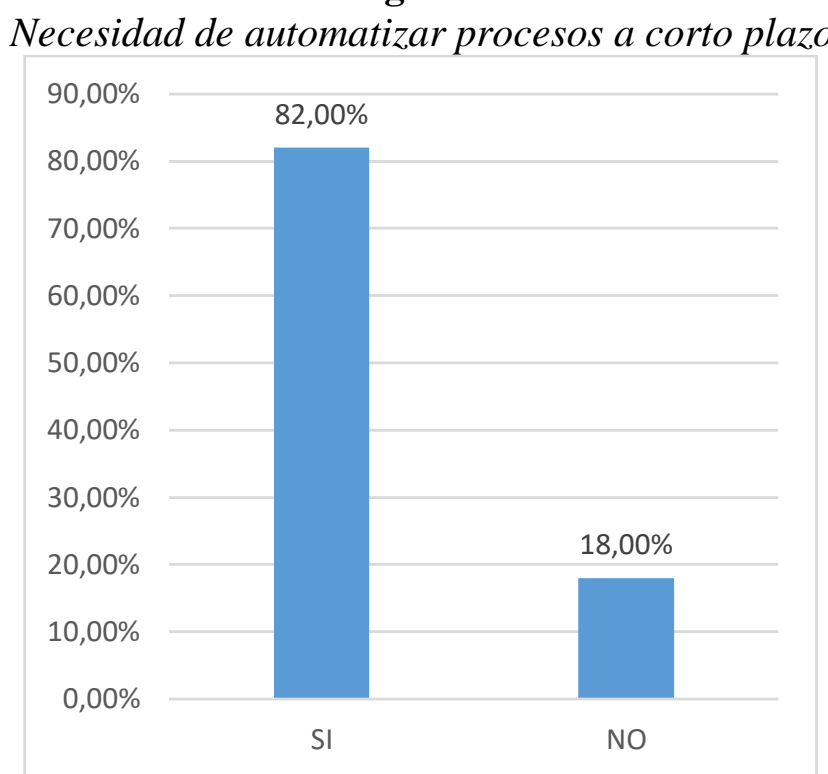

Fuente: Encuesta a empleadores, 2020

Se puede observar que casi todos los encuestados manifiestan que en un futuro de corto plazo necesitarán procesos informatizados para mejorar el procesamiento $\mathrm{y}$ almacenamiento de la información.

La figura 4 presenta la frecuencia con la que se presentan problemas de software en los diferentes departamentos de las organizaciones.

\section{Figura 4}

Problemas de software por departamento

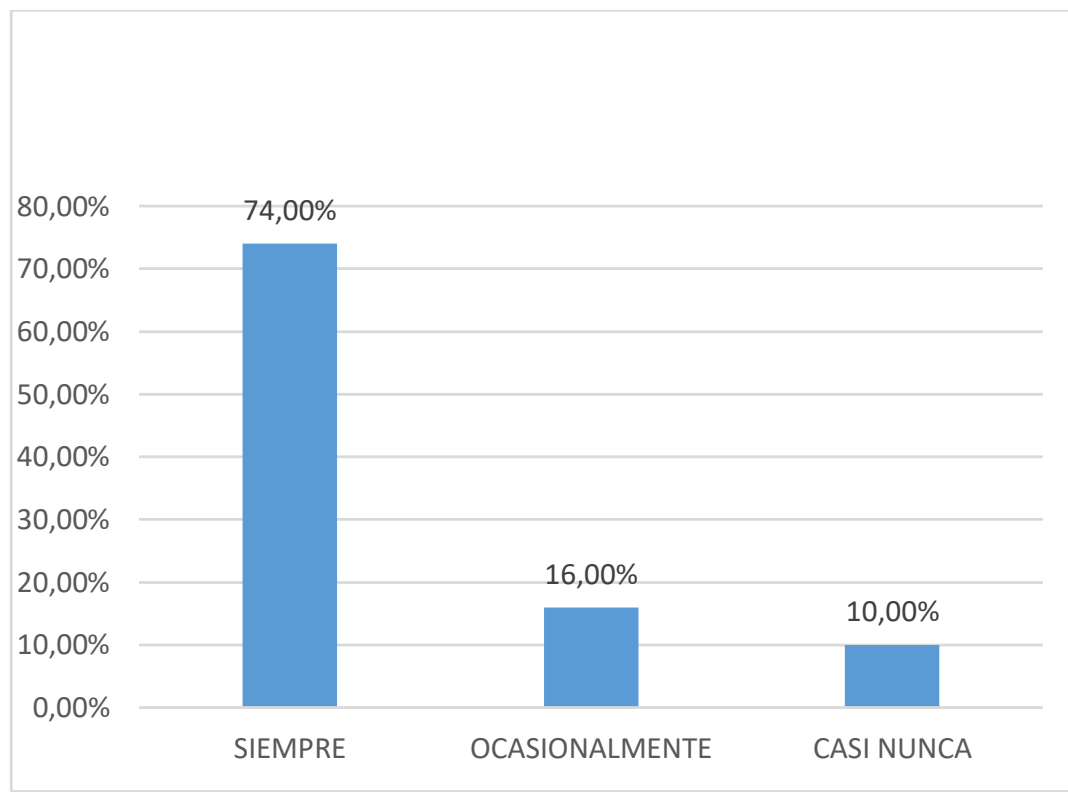

Fuente: Encuesta a empleadores, 2020

Se puede constatar que más de la mitad de los encuestados señalan tener problemas recurrentes en el uso de software al no tener personal capacitado en el área, provocando demoras en los procesos institucionales. 
En la figura 5 muestra la necesidad de personal con conocimientos suficientes para resolver problemas de software.

\section{Figura 5}

Personal con conocimientos suficientes para resolver problemas de software

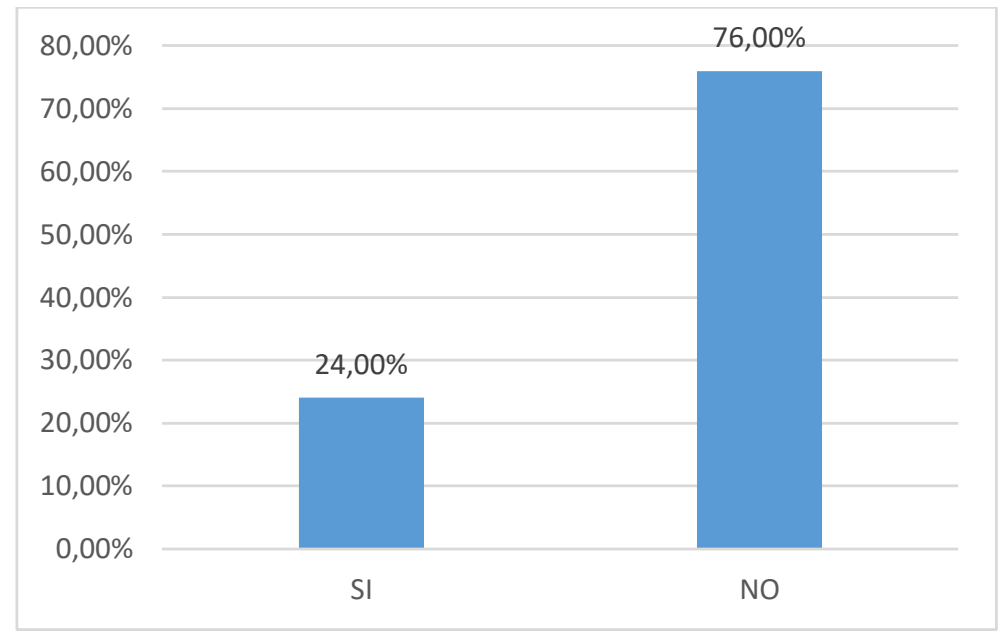

Fuente: Encuesta a empleadores, 2020

La mayoría de las instituciones no cuentan con personal calificado para resolver problemas referentes al software, o que desarrollen sistemas que permita automatizar los procesos repetitivos que realizan diariamente.

La figura 6 refleja la aceptación del profesional en Ingeniería de Software para laborar en diferentes organizaciones.

\section{Figura 6}

Aceptación del profesional en Ingeniería de Software para laborar en diferentes instituciones

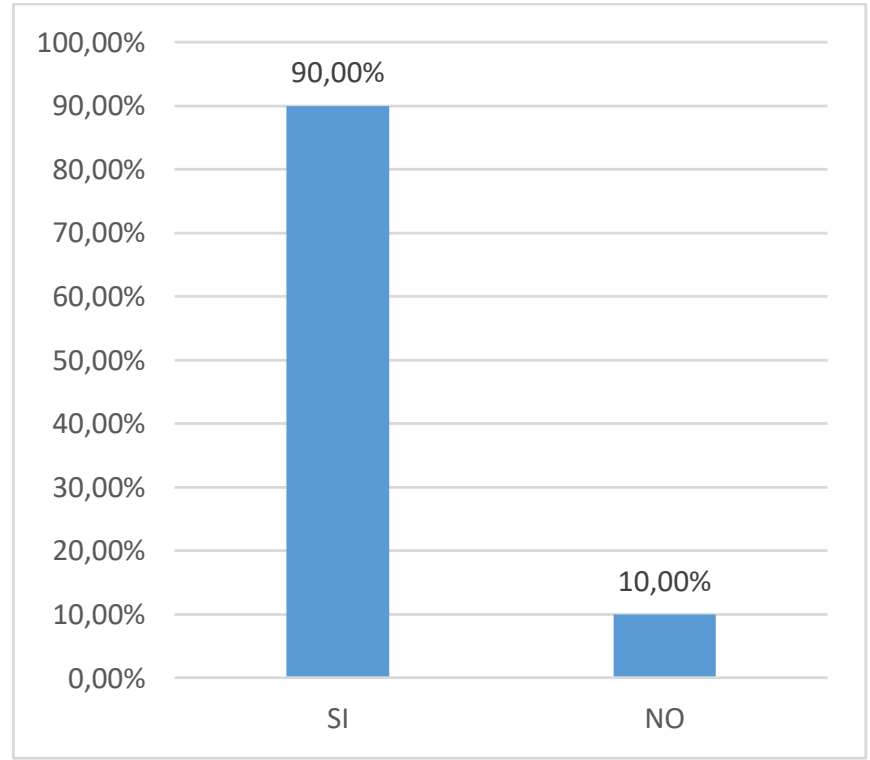

Fuente: Encuesta a empleadores, 2020 
La mayoría de los empleadores encuestados estarían dispuestos a contratar Ingenieros(as) en Software graduados en la Universidad Estatal de Bolívar, porque estos profesionales conocen la realidad de su contexto y pueden ayudar a mejorar la mismas.

Existen varios estudios relacionados con la pertinencia de carreras, como el realizado por Martínez \& Mendoza (2017), denominado "Pertinencia de la carrera en pedagogía de la actividad física y deporte de la Universidad Central del Ecuador" en el cual evidencian la pertinencia de la carrera con las necesidades del contexto nacional, regional y local, basados en una exhaustiva revisión documental de reglamentos, literatura especializada e informes de organismos nacionales e internacionales y publicaciones científicas de las de nuevas tendencias metodológicas, tecnológicas y del conocimiento, además de una investigación de campo donde utilizaron cuestionarios para recoger información de forma directa de estudiantes, profesionales, expertos, representantes de los sectores estratégicos gubernamentales, e instituciones relacionadas con la profesión.; similar a la metodología empleada para el análisis de la pertinencia del currículo de la Carrera de Software en la UEB, que permitió valorar y reestructurar los campos de formación curricular con una visión moderna acorde a las necesidades de la sociedad actual.

En el estudio de López (2016), titulado: “ Análisis de la pertinencia de la ingeniería en las TIC del ITIC de Tepic desde la perspectiva de los empleadores", en el que principalmente determina el perfil de egreso de los futuros profesionales, obteniendo la evaluación de la calidad del plan de estudios, su productividad, demanda y necesidades a las que responde; semejante a la investigación realizada en la carrera de Software de la UEB en la que se determina la necesidad de mejorar el currículo de la carrera para entregar a la sociedad profesionales integrales en la ingeniería de software que aporten al crecimiento de la empresa pública y privada.

Por otra parte García et al. (2014), en su investigación titulada "Estudio de pertinencia de la Carrera de Ingeniería Ambiental para la Región 5", se asemeja con este estudio al momento del cálculo de las muestras y en el diagnóstico realizado a las instituciones públicas y privadas para conocer la demanda profesional; a la vez difiere con esta investigación, pues no se analiza la opinión de los estudiantes de bachillerato, siendo importante para la presente investigación conocer la perspectiva de los potenciales aspirantes a la carrera de Software.

El "Proyecto Educativo del Programa - Ingeniería en Sistemas e Informática", desarrollado por Universidad Nacional de Colombia (2013), describe la importancia de una carrera en el ámbito de la informática, pues la tecnología dinamiza e integra prácticamente a todos los procesos de la vida moderna, en empresas productoras de bienes y servicios sean del sector público o privado. Lo que genera una demanda continua de profesionales preparados para diseñar, desarrollar, gestionar y controlar aplicaciones informáticas y sistemas relacionados, siendo importante mantener actualizado el plan de estudio de la carrera para solventar las necesidades actuales del contexto. 


\section{Conclusiones}

- La carrera de software muestra componentes educativos concretos dentro de su currículo, sin embargo, es importante realizar una actualización a los mismo para mejorar las habilidades y capacidades de los futuros ingenieros, considerando aspectos prácticos y técnicos de la ingeniería del software, lo que contribuirá a un favorable desenvolvimiento profesional.

- Los profesionales de la carrera podrán aportar con aplicaciones software de calidad en diversas áreas de la sociedad, siendo un aporte al desarrollo de la matriz productiva.

- La Universidad Estatal de Bolívar se convierte en un referente de la carrera de Software en la región sierra de la zona 5, evidenciando su pertinencia, considerando que sólo dos universidades de la región costa de esta zona ofertan la carrera.

- Es importante realizar más charlas del sistema educativo universitario en las unidades educativas y/o colegios, específicamente resaltando las competencias y habilidades del futuro Ingeniero(a) en Software, considerando que existe una alta aceptación de la carrera por los estudiantes de educación secundaria.

\section{Referencias Bibliográficas}

Asociación Nacional de Universidades e Instituciones de Educación Superior [ANUIES]. (s.f.). "Acerca de la Anuies". http://www.anuies.mx/anuies/acerca-de-la-anuies (19-07-2020).

Asamblea Nacional de la República del Ecuador. (2010). Ley Orgánica de Educación Superior. Registro Oficial No. 298, 12 de octubre. Quito.

Bologna, E. (2018). Métodos estadísticos de investigación. Editorial Brujas. https://elibro.net/es/ereader/bibliotecaueb/106355?page $=159$

Consejo de Aseguramiento de la Calidad de la Educación Superior [CACES]. (2017). Modelo Genérico de Evaluación del Entorno de Aprendizaje de Carreras en Ecuador. Quito.

Dirección de Planeamiento y Aseguramiento de la Calidad de la UEB. (2020). Estudio Prospectivo de la Carrera de Software. Guaranda

García G., Bastidas M., Pacheco F., y Andrade M., (2014). Estudio de pertinencia de la Carrera de Ingeniería Ambiental para la Región 5, Revista Ciencia UNEMI, (2), 69-80.

Goyes J., Alvarado E., Arguello A., Gavilánez V., Pinto M. y Dávila M. (2021). Análisis Descriptivo de la Educación Superior en el Ecuador. Dirección de Planeamiento y Aseguramiento de la Calidad UEB. Guaranda. 
López, C. (2016). Análisis de la pertinencia de la ingeniería en las TIC del ITIC de Tepic desde la perspectiva de los empleadores. Revista Electrónica sobre Educación Media y Superior, 3(6), 1-20.

Martínez J. y Mendoza M., (2017). Pertinencia de la carrera en pedagogía de la actividad física y deporte de la Universidad Central del Ecuador. Revista Digital de Educación Física, (45), 81-92.

Ministerio de Educación. (2019). Archivo Maestro de Instituciones Educativas (AMIE) 2018-2019. Ecuador

Secretaría de Educación Superior, Ciencia, Tecnología e Innovación [SNIESE]. (2020). Información actualizada sobre la educación superior del Ecuador.

Secretaría Técnica del Sistema Nacional Descentralizado de Planificación Participativa [SENPLADES]. (2017). Zona de Planificación 5 - Litoral. Quito.

Tamayo M. y Cerda M. (2017). El Rol de la pertinencia en la evaluación de carreras universitarias en el Ecuador. Revista Electrónica sobre Ciencias Pedagógicas e Innovación.

Organización de las Naciones Unidas para la Educación, la Ciencia y la Cultura [UNESCO]. (1995). Documento sobre Políticas para el cambio y el desarrollo de la Educación Superior. Caracas.

Organización de las Naciones Unidas para la Educación, la Ciencia y la Cultura [UNESCO]. (1998). Conferencia Mundial sobre Educación Superior, La educación Superior en el Siglo XXI: visión y acción. Informe Final. París.

Universidad Autónoma de Yucatán [UADY]. (2012). Guía para elaboración de estudios de pertinencia social, factibilidad, y estado del arte para la creación, modificación y liquidación de programas educativos. México: Dirección General de Desarrollo Académico.

Universidad Nacional de Colombia. (2013). Proyecto Educativo del Programa Ingeniería de Sistemas e Informática. Medellín.

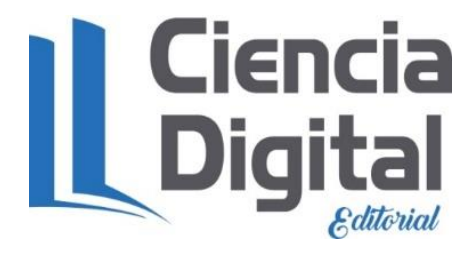




\section{PARA CITAR EL ARTÍCULO INDEXADO.}

García Camacho, G. I., Espín Morejón, M. A., Bonilla Manobanda, M. E., \& Carrión Buenaño, D. P. (2021). Pertinencia curricular de la carrera de Software en la Universidad Estatal de Bolívar. ConcienciaDigital, 4(4.2), 6-21. https://doi.org/10.33262/concienciadigital.v4i4.2.1933

\section{¿Ciencia}

El artículo que se publica es de exclusiva responsabilidad de los autores y no necesariamente reflejan el pensamiento de la Revista Conciencia Digital.

El artículo queda en propiedad de la revista y, por tanto, su publicación parcial y/o total en otro medio tiene que ser autorizado por el director de la Revista Conciencia Digital.

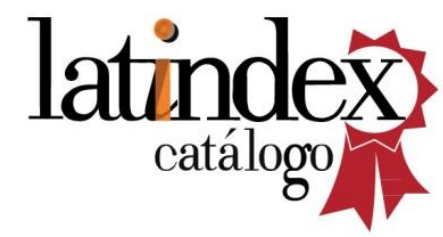

